\title{
Enseignement culturel de la religion et édification de la modernité
}

Le cas du Québec

Cultural teaching of religion and building modernity. The case of Quebec

Enseñanza cultural de la religión y edificación de la modernidad. El caso de

Québec

\section{Louis LeVasseur}

\section{OpenEdition}

\section{Journals}

Édition électronique

URL : http://journals.openedition.org/ries/3499

DOI : 10.4000/ries.3499

ISSN : 2261-4265

Éditeur

Centre international d'études pédagogiques

Édition imprimée

Date de publication : 1 septembre 2013

Pagination : 123-132

ISBN : 978-2-85420-600-5

ISSN : $1254-4590$

Référence électronique

Louis LeVasseur, «Enseignement culturel de la religion et édification de la modernité », Revue internationale d'éducation de Sèvres [En ligne], 63 | septembre 2013, mis en ligne le 01 septembre 2015, consulté le 01 mai 2019. URL : http://journals.openedition.org/ries/3499; DOI : 10.4000/ries.3499 


\section{Enseignement culturel de la religion et édification de la modernité}

\section{Le cas du Québec}

\section{Louis LeVasseur}

En procédant au remplacement des commissions scolaires confessionnelles par des commissions scolaires linguistiques en juillet 1998, la question de l'enseignement religieux au Québec n’a pas été réglée pour autant. Il subsiste beaucoup de réticences, chez certains groupes sociaux à tout le moins, face à la présence du religieux dans l'espace public et à l'école. Le Québec est traversé par des orientations culturelles en tension qui pourraient en partie expliquer son ambivalence face à l'enseignement de la religion. Les Québécois se veulent ouverts à la diversité culturelle et religieuse, sont hostiles à une vision strictement instrumentale du monde mais ils craignent une résurgence du religieux qui serait contraire à l'idée qu'ils se font de la modernité et qui pourrait compromettre certaines valeurs auxquelles ils sont attachés : autonomie de la personne, reconnaissance de la spécificité et de la liberté de l'enfant, laïcité, vision égalité hommes-femmes, rationalité scientifique, etc. Cependant, l'enseignement de la religion au Québec va-t-il nécessairement à contresens de la modernité qui tend à s'édifier depuis la Révolution tranquille ${ }^{1}$ ? Et comment un tel enseignement peut-il s'inscrire dans un processus de modernisation généralement identifié à une sécularisation et à une rationalisation qui conduisent au retrait graduel du religieux ? Que disent les enseignants à cet égard ?

\section{UN NOUVEAU PROGRAMME D'ENSEIGNEMENT CULTUREL De LA RELIGION AU QuÉBeC ${ }^{2}$}

La tentative la plus concrète visant à concilier l'enseignement de la religion avec les cadres d'une société moderne et pluraliste provient du Groupe de travail sur la place de la religion à l'école (MÉQ, 1999) dont le rapport (rapport Proulx) a conduit à l'adoption du programme d'Éthique et culture religieuse (MELS, 2007a), lequel repose sur deux principes politiques fondamentaux visant à baliser le rôle de l'État au regard de l'enseignement de la religion. Le premier principe est celui de la démocratie libérale: «Le Québec est une démocratie

\footnotetext{
1. Mouvement qui correspond à la modernisation accélérée de la société québécoise à partir de 1960 à environ 1966. 2. Pour un historique plus complet de l'enseignement de la religion au Québec, voir le mémoire de maîtrise de Stéphanie Tremblay, publié en 2011.
} 
libérale qui doit, dans tous les domaines, respecter la norme de l'égalité de tous les citoyens » (MÉQ, 1999, 77). Ceci signifie concrètement que tous les élèves doivent avoir droit aux mêmes services - et non les élèves catholiques à un enseignement confessionnel ou moral et les autres élèves à un enseignement moral seulement. Le second principe est celui de la "nécessaire neutralité de l'État en matière de religion »(MÉQ, 1999, 79). Il est recommandé que le ministère adhère à un principe de neutralité égalitaire cependant distinct de ses deux principaux modèles : le communautarien, que l'on retrouve fréquemment aux États-Unis, et le républicain, dominant, que l'on retrouve en France. Le premier conduit à un pluralisme mono-ethnique ou religieux, dont le principe menace d'éclatement l'espace public et qui ne favorise pas le vivre-ensemble. Quant au second modèle, qui consiste à refuser toute manifestation religieuse dans les espaces publics, il est rejeté d'emblée par le rapport Proulx, dont les auteurs estiment que le but de l'enseignement consiste non pas à éluder le fait religieux dans l'école mais à le reconnaître sans pour autant s'engager dans la voie de la confessionnalité ni même de la spiritualité. Quelle importance, alors, de maintenir l'enseignement religieux à l'école et comment l'y maintenir tout en contournant la question de la confessionnalité et de la foi ?

La première question touche à la pertinence de l'enseignement de la religion à l'école. La reconnaissance du fait religieux comme composante de la culture contemporaine justifierait à elle seule la présence de l'enseignement de la religion à l'école. Mais il y a plus. L'école québécoise s'est donné pour mission la socialisation de tous les élèves et doit, en ce sens, favoriser tout à la fois la construction identitaire des élèves, éviter l'exclusion sociale et favoriser le vivre-ensemble. Le rapport Proulx adhère en bonne partie à l'idée de neutralité d'une école laïque (MELS, 2007a, 192-197) pour tous mais lui préfère l'option d'un enseignement culturel des religions car « elle est cohérente par rapport aux buts sociétaux qui consistent en la construction d'un espace civique commun et en la socialisation des jeunes au sein d'une société pluraliste et valorisant la diversité des patrimoines religieux » (MELS, 2007a, 214). Quant au philosophe et analyste du programme, Georges Leroux, il convient, pour des raisons analogues, d'éviter de calquer le modèle républicain français :

«(...) sous la pression du pluralisme culturel et de l'immigration, ce modèle [modèle républicain à la française] révèle son incapacité à intégrer dans l'espace public les communautés, surtout celles issues de pays musulmans, qui sont en déficit d'identité et qui se recomposent maintenant dans les franges politiques de l'islamisme ou de mouvements sectaires divers. Le mémoire présenté en 2002 par le philosophe Régis Debray au président de la République ne laisse aucun doute sur les orientations à prendre : il est plus que temps d'introduire un enseignement culturel des traditions religieuses et des cultures constitutives de l'Europe, faute de quoi les jeunes Français manqueront de cette culture morale et religieuse non seulement pour comprendre les autres, mais pour accéder à leur propre expérience. »(Leroux, 2007, 61) 
La seconde question, une fois reconnue la pertinence des cours de religion dans le cursus, touche à la formule à retenir pour l'enseignement. Une douzaine de formules possibles ont été étudiées et une a été retenue qui permet de concilier les principes politiques de démocratie libérale et de neutralité égalitaire telle que définie précédemment mais également les finalités de socialisation de l'école québécoise. Conséquemment, l'enseignement de la religion à l'école ne sera plus confessionnel mais culturel. Il s'agit d'un enseignement permettant de réunir tous les élèves dans un même espace, afin de comprendre les phénomènes religieux dans la perspective objectiviste des sciences humaines. Il s'agit donc moins de transmettre les fondements de la foi ou de favoriser l'éveil de celle-ci que de montrer les grands principes structurant les grandes religions du monde, y compris les religions amérindiennes. Le programme (MELS, 2007a) qui découlera des réflexions du rapport Proulx (MÉQ, 1999) sera lui-même structuré autour de trois principales compétences : réfléchir sur des questions éthiques, manifester une compréhension du phénomène religieux et pratiquer le dialogue. Aucune de ces compétences ne touche donc à la croyance ou à la foi de l'élève et la troisième renvoie à l'idée de démocratie et de pluralisme, qui sont au cœur d'une conception contemporaine du bien commun auquel Habermas (1988) associe la rationalité délibérative et l'intersubjectivité. La rationalité délibérative doit donc permettre aux élèves de discuter, de débattre, et surtout, de fonder les termes d'une société dont les valeurs sont moins le fait d'un héritage que d'un projet rendu possible par la critique et la délibération (MÉQ, 1999, 89). En ce sens, le programme d'Éthique et culture religieuse est beaucoup moins l'expression d'une vision traditionnelle, religieuse ou métaphysique que moderne et rationnelle du monde.

\section{LA FIN DE LA RELIGION EN OCCIDENT OU LA RELIGION, MOTEUR DE LA MODERNITÉ ?}

L'enseignement de la religion au Québec, selon ce qu'en disent les textes prescriptifs (MÉQ, 1999 ; MELS, 2007a) et analytiques (Leroux, 2006 ; Tremblay, 2011) qui s'y rapportent, serait moderne en raison de son approche objective, et non plus confessionnelle, son pluralisme et son insistance sur le développement des compétences délibératives des élèves dans un espace public commun, celui d'une classe composée d'élèves aux appartenances morales et religieuses différentes. Il nous semble cependant que le discours des enseignants révèle une dimension subjective de l'enseignement, dont la modernité diffère partiellement de la précédente. En effet, la modernité peut s'interpréter comme étant une disposition culturelle propre à l'Occident, qui émerge à la Renaissance et que traduisent admirablement Descartes au XVII ${ }^{\mathrm{e}}$ siècle et Kant au XVIII ${ }^{\mathrm{e}}$ siècle, chacun à leur manière faisant du sujet (et de sa conscience) l'instance de légitimation des normes et des valeurs. L'instance de légitimation de la croyance 
religieuse passerait-elle, pour les enseignants de notre recherche, d'une instance transcendante au sujet? Insistons ici sur le fait que notre article ne porte pas sur le rapport à la croyance des élèves eux-mêmes mais bien sur l'idée d'un éventuel établissement, par les enseignants, d'un rapport subjectif à la croyance chez les élèves de toutes confessions, y compris non chrétiennes. La question n'est donc pas de savoir si les élèves développement réellement des dispositions culturelles modernes par l'entremise d'un enseignement ainsi orienté vers un processus de subjectivation du rapport à la croyance, mais bien si les enseignants disent chercher à le faire.

Avant de procéder à l'analyse de ce discours, montrons en quoi le rapport au religieux de bien des croyants dans nos sociétés contemporaines est susceptible d'être moderne.

L'explication surnaturelle de l'ordre du monde est certes incompatible avec la modernité (Habermas, 1988), mais pas nécessairement la désinstitutionnalisation de la religion et la manière dont les croyants tendent à vivre leur foi de manière subjective, loin de l'orthodoxie des grandes confessions. Dans la mesure où la croyance tend à s'individualiser et à se «subjectiver », elle pourrait tout à fait constituer une ressource, parmi bien d'autres, par laquelle l'individu se définit de manière autonome sur le plan identitaire.

En ce sens, la définition canonique que Durkheim a donnée de la religion ne recouvre plus l'ensemble des rapports au religieux aujourd'hui. Pourquoi référer ici à cette définition de Durkheim ? Parce qu'elle montre au mieux le rôle de socialisation et d'intégration que jouait la religion dans une modernité que nous qualifions ici de classique; elle permet de voir, par contraste, que le rôle de la religion dans notre modernité avancée, tout comme les modes de socialisation, a changé. Selon Durkheim, la religion constitue un facteur d'intégration au sein de la communauté et sans cette dimension communautaire qu'il lui reconnaît, il ne saurait y avoir de religion :

"Une religion est un système solidaire de croyances et de pratiques relatives à des choses sacrées, c'est-à-dire séparées, interdites, croyances et pratiques qui unissent en une même communauté morale, appelée Église, tous ceux qui y adhèrent. Le second élément qui prend ainsi place dans notre définition n'est pas moins essentiel que le premier; car, en montrant que l'idée de religion est inséparable de l'idée d'Église, il fait pressentir que la religion doit être une chose éminemment collective. » (Durkheim, 2008, 65)

Dans L'Évolution pédagogique en France, Durkheim $(1990,27)$ présentera l'Église comme un lieu de diffusion de vie intellectuelle et définira le christianisme en tant que "religion idéaliste, un système d'idées, un corps de doctrines » dont la transmission suppose l'enseignement et la culture (Durkheim, 1990, 30). Cette manière de concevoir la religion comme étant une doctrine institutionnalisée auquel adhère le croyant, qui induit chez lui une manière de voir le monde et des pratiques codifiées, continue très certainement aujourd'hui 
de traduire une forme de la vie religieuse caractéristique de plusieurs croyants. Mais n'y a-t-il pas d'autres manières de vivre sa foi, plus individualisées, plus autonomes, moins tributaires du respect du dogme et plus proches d'une expérience affective ou de ce que l'on pourrait appeler l'expérience vécue ${ }^{3}$ ? C'est à tout le moins l'hypothèse qu'il est possible de faire en suivant les réflexions de spécialistes de la forme du religieux dans nos sociétés contemporaines. Le sociologue des religions, Jean-Paul Willaime $(1995,109)$ affirme ce qui suit, afin de caractériser «l'incertitude ultramoderne de la religion »:

«En réaction contre les froideurs de la rationalité instrumentale, le religieux est réinvesti comme émotion et subjectivité et s'éprouve dans l'expérience. La dérégularisation institutionnelle du religieux et la déstabilisation de son langage, le divorce entre les représentations et organisations religieuses produisent des signifiants religieux flottants, des signifiants qui ne sont plus arrimés à des systèmes stabilisés de signification. À travers l'expérience, par l'authentification du vécu, on redonne sens à des signifiants, mais de façon instable et précaire. » (Willaime, 1995, 109)

Charles Taylor a pour sa part réalisé un essai sur «La diversité de l'expérience religieuse aujourd'hui » et plus spécifiquement sur le rapport que William James, représentant du pragmatisme philosophique aux États-Unis dans la seconde moitié du XIX ${ }^{\mathrm{e}}$ siècle, entretenait aux sciences et à la religion. Selon James, la science triomphante de son époque avait tort de nier l'existence d'une expérience religieuse pouvant constituer une "vérité " pour le croyant, et afin d'accéder à cette expérience religieuse, le croyant devait à la fois se déprendre de ses appartenances ecclésiales et individualiser son rapport à Dieu :

"Ainsi le vrai foyer de la religion, c'est la conscience individuelle et non le corps ecclésial. C'est là une facette de la thèse de James. L'autre, c'est que la vraie religion se situe dans l'expérience, c'est-à-dire dans le sentiment, et non dans les formulations que les gens élaborent pour définir, justifier et rationaliser leurs sentiments (opérations qui, bien sûr, sont fréquemment entreprises par les églises).» (Taylor, 2003, 13)

Ailleurs, Taylor mentionne que "l'expérience religieuse, au sens où James l'entend, (évite) les liens collectifs et la surthéorisation » (Taylor, 58) et il cite le mot de James selon lequel «quand une religion devient orthodoxe, elle perd à tout jamais son intériorité » (James, cité dans Taylor, 2003, 12).

Où se situent les enseignants ayant participé à notre recherche par rapport à l'appartenance collective inhérente à la religion selon Durkheim ou à la subjectivation de l'expérience religieuse selon Willaime, Taylor et James dans nos sociétés contemporaines? Les préoccupations pour le collectif sont-elles définitivement absentes de leurs propos? De quelle manière peut-on voir que 
la modernité est en construction à travers leur enseignement, alors que la religion est généralement associée à une vision du monde en tension avec la modernité ?

\section{QUELQUES INDICATIONS MÉTHODOLOGIQUES}

La recherche a été menée avec 19 enseignants de religion au secondaire au printemps 2008, soit quelques mois avant l'implantation du nouveau programme d'Éthique et culture religieuse (MELS, 2007a) en automne $2008^{4}$. Insistons cependant sur le fait que les enseignants connaissaient déjà tous ce nouveau programme, avaient suivi une formation à son sujet, que plusieurs enseignants disaient déjà enseigner conformément aux objectifs et au contenu de ce programme et que notre entretien était construit de manière telle qu'il pouvait s'adresser aux enseignants indépendamment du programme alors en vigueur ou de celui à venir. Par exemple, nous avons posé des questions sur la place de la religion à l'école, sur la formule pédagogique pertinente dans une société pluraliste et multi-religieuse, sur la mission de socialisation de l'école québécoise et sur la contribution de l'enseignement de la religion à cette mission. Une question particulière touchait à la construction identitaire, soit du collectif, soit de l'individu dans l'enseignement. Les entretiens ont eu lieu également dans les villes de Montréal et de Québec. L'échantillonnage était composé de douze hommes et de sept femmes. On y trouvait des enseignants en début, en milieu et en fin de carrière. Nous n'avons pas demandé aux enseignants de nous indiquer s'ils étaient ou non croyants ou pratiquants ${ }^{5}$. Une fois les entrevues retranscrites, nous avons procédé à une analyse de contenu, les propos des enseignants ayant été regroupés par thèmes. Les deux principaux thèmes retenus pour les fins d'analyse dans ce présent article touchent à la subjectivation du rapport à la religion chez l'élève et à la question du lien social dans une société diversifiée tant culturellement que religieusement.

\section{QUE DISENT LES ENSEIGNANTS AU SUJET DE L'ENSEIGNEMENT DE LA RELIGION?}

Deux postures fortes se dégagent de nos entrevues, et qui insistent chacune sur des aspects individuels et collectifs reliés à l'enseignement de la religion. L'élève doit être libre de se former une opinion face à la religion et il

\footnotetext{
4. Mentionnons que l'implantation de ce programme ne s'est pas faite sans heurts. Dès la publication du rapport Proulx (MÉQ, 1999), des voix se sont élevées afin de dénoncer l'« intrusion » de l'État en éducation, qui bafouerait la liberté de choix des parents. Dans certaines villes « mono-ethniques » ou « mono-religieuses », des parents estiment que leurs enfants ont droit à un enseignement confessionnel.

5. Le fait qu'un enseignant soit pratiquant ne signifie pas nécessairement qu'il s'oppose à un enseignement culturel de la religion, pas plus que les enseignants d'histoire ayant des convictions nationalistes ne s'opposent nécessairement à l'enseignement d'une histoire pluraliste et ouverte (LeVasseur et Cardin, 2012).
} 
doit pouvoir discuter librement de phénomènes religieux avec les autres élèves. Et même, certains enseignants ne pensent pas indépendamment l'un de l'autre ces aspects subjectifs et dialogiques.

\section{La subjectivation du rapport à la religion}

L'idée d'une forte subjectivation du rapport à la religion est décelable dans à peu près tous les propos des enseignants, qu'ils soient de Montréal ou de Québec, en début ou en fin de carrière, y compris ceux qui enseignent dans les écoles privées dont certaines peuvent conserver un lien avec le projet d'une école confessionnelle. Les enseignants disent alors insister moins sur la dimension religieuse de la religion, donc pourrions-nous dire ici, sur l'éveil de la foi et de la croyance dans un cadre orthodoxe, prescriptif et institutionnalisé, que sur la dimension éthique ou spirituelle de la croyance, ce qui renvoie à l'idée d'une certaine indépendance du croyant face à la confession religieuse et à son orthodoxie. À la limite, se décèle dans les propos des enseignants la volonté de favoriser un rapport non plus collectif mais subjectif à la croyance. La croyance n'est plus communautaire mais individuelle. À la question de savoir si l'enseignement va dans le sens de la formation d'une identité collective (au sens de communauté de croyants) ou d'identités individuelles, voici les réponses d'enseignants presque choisies au hasard, tant cette tendance à individualiser le rapport à la foi est récurrente dans le discours de tous les enseignants :

"Ça va être la formation de l'individu. Il y a très peu d'enseignants, je pense, qui travaillent encore pour la gloire du Christ. Ils n'ont pas l'impression de fabriquer ou de contribuer à la croissance de jeunes dans l'Église. » (Sujet 1) "Je fais en sorte de favoriser une réflexion et même une prise de position, un questionnement, de réveiller la conscience dans mon enseignement, mais je prends quand même une distance parce que c'est de l'enseignement, ce n'est pas de l'évangélisation. Il y a une distance qui laisse à l'élève sa liberté de penser. »(Sujet 2)

"Je pense que c'est une formation individuelle. L'identité collective, ça, c'est terminé. D’ailleurs, c'est pour ça qu'on appelle le cours Éthique et culture religieuse. Ce n'est plus pour le fondement collectif, c'est pour le fondement individuel : avoir une ouverture pluraliste par rapport aux valeurs des autres, aux autres religions. Donc, c'est le fondement individuel et l'élève va trouver, autant dans le christianisme que dans l'islam ou dans le judaïsme, il va trouver des valeurs qui l'interpellent dans son identité.» (Sujet 4)

«En premier, ce que je vise, c'est la formation de la personne. Toute la réflexion éthique peut vraiment être un élément important dans la formation personnelle du jeune. L'identité collective, je ne pense pas qu'on sort du cours en disant: "Nous, les Québécois catholiques, etc." ». (Sujet 5)

Dans tous les extraits d'entrevue que nous venons de citer, la question de la formation d'une identité collective semble reléguée aux oubliettes. Il faut 
cependant tenir compte des effets des questions sur l'importance relative que les enseignants accordent à certains éléments plutôt qu'à d'autres. Car dans plusieurs extraits d'entrevue, les enseignants montrent leur préoccupation pour la dimension collective reliée à l'enseignement de la religion. Mais cette dimension collective est-elle analogue à ce que Durkheim $(2008,65)$ entendait par « communauté morale », soit l'Église?

\section{Une formation au dialogue favorisant la recomposition de l'identité collective}

Les enseignants font référence dans leur discours non pas à un corps moral mais à un corps délibératif, où la rationalité et la critique occupent une place aussi centrale que la foi et la croyance dans la religion :

"Mes cours sont orientés autour du vivre-ensemble. Mes approches, mes méthodes, mes interventions, mes attitudes font en sorte (...) que chaque individu puisse s'exprimer en se confrontant aux autres dans une perspective de dialogue. Il ne faut pas oublier une chose fondamentale dans le nouveau programme, c'est que ce qui lie tous les éléments de son contenu, en éthique, en religion, c'est la compétence au dialogue. Elle est vraiment centrale ; elle est le pivot, le pilier, du nouveau programme. »(Sujet 12)

Pour l'enseignant suivant, la culture publique commune doit même prédominer sur les croyances religieuses, à tout le moins, celles qui seraient incompatibles avec les valeurs collectives :

«Si la croyance va à l'encontre des valeurs collectives, qui est le respect des femmes, le respect de l'autre, c'est là qu'il peut y avoir une tension entre les deux. Alors, c'est peut-être cette logique-là, essayer de faire comprendre que "voici vous avez vos croyances, c'est correct», mais il faut que ça aboutisse au respect de l'individu, des minorités, des homosexuels, des femmes. Sinon, il faut se questionner. »(Sujet 10)

On le voit à ce qui précède, contrairement à un enseignement confessionnel de la religion, où il s'agit de transmettre un héritage spirituel, les enseignants conçoivent leur enseignement comme un instrument au service d'une culture publique en tant que projet. Il s'agit moins pour l'élève de croire et de communier dans un contexte d'unité spirituelle que de débattre dans celui d'un pluralisme en tension avec comme horizon le possible établissement d'un consensus.

\section{L'ENSEIGNEMENT DE LA RELIGION, SYMPTÔME D'UNE MODERNITÉ INCOMPLÈTE ?}

Nous avons mentionné, dès notre introduction, que l'enseignement de la religion au Québec pouvait constituer un moteur de la modernité. En quoi, alors, la modernité, voire la double modernité d'un tel enseignement 
consiste-t-elle ? Dans la mesure où nous définissons la modernité comme étant la période de l'histoire où l'individu cherche à conquérir son autonomie et où l'ensemble des individus parviennent à créer les conditions du vivre-ensemble sans s'en remettre à des forces surnaturelles qui auraient le pouvoir de se soustraire à la volonté ou à la conscience du et des sujets, la modernité des enseignants québécois de la religion consiste dans la recherche de l'autonomie de la pensée morale des élèves et, également, dans la dimension délibérative de l'enseignement arrimé à l'idée de consensus. Consensus qui n'aurait plus rien de substantiel, encore moins de métaphysique ou de surnaturel. Cependant, quelle que soit la valeur d'un tel enseignement, le fait d'évoquer son éventuelle modernité ne suffit pas à lui éviter toute critique. Nous en formulerons deux principales, par lesquelles nous discuterons la place que cet enseignement occupe au sein du Programme de formation de l'école québécoise (MELS, 2007b) et, paradoxalement, au sein même de la modernité.

Premièrement, la religion peut être instrumentalisée et constituer un moyen permettant aux élèves de se réunir et de discuter ensemble de leurs convictions - quitte à ce que celles-ci ne soient pas ancrées dans la croyance -, et du devenir collectif - quitte à ce que celui-ci ne soit aucunement encastré dans une vision religieuse totalisante du monde. Mais si la finalité de cet enseignement devient le développement des compétences délibératives, ce qui doit comprendre l'ouverture, la tolérance, la critique, l'argumentation, et également l'idée de consensus afin d'échapper aux pièges du relativisme culturel qui compromet le débat sur la question des valeurs (MÉQ, 1999, 91), n’aurait-on pu remplacer l'enseignement de la religion par celui de l'histoire, des arts et de la littérature, afin d'amener les élèves à discuter entre eux de leur appartenance et de leurs convictions?

Deuxièmement, et nous suivons de près ici les réflexions du philosophe Alain Renaut (2010, 184), comment concilier, dans nos sociétés modernes, l'essence même de la religion avec la subjectivation de la culture ? Fondamentalement, la religion ne suppose-t-elle pas que le sens du monde appartienne, en dernière instance, à un autre qui se trouve, par le fait même, déposséder le sujet de son autonomie morale? En quoi consiste alors la pertinence d'une telle conception du monde, où l'homme est assujetti à une force qui le dépasse, dans un curriculum, celui de l'école québécoise, intégralement fondé sur l'autonomie intellectuelle, morale et esthétique du sujet (LeVasseur, 2012) ? Qui plus est, la démocratie elle-même est-elle pensable sur fond d'une pensée (religieuse) qui reconduit, fondamentalement, le principe d'hétéronomie (Renaut, 2010, 171) ?

\section{Bibliographie}

DURKHEIM É. (2008): Les formes élémentaires de la vie religieuse. Paris: Presses universitaires de France.

HABERMAS J. (1988) : Le discours philosophique de la modernité. Paris : Gallimard. 
LEROUX G. (2007) : Éthique, culture religieuse, dialogue. Montréal : Fides.

LEVASSEUR L. (2012) : "L'école québécoise et la culture scolaire : développement intégral de l'enfant, développement cognitif de l'élève et contextes éducatifs". Phronesis, vol. 1, $\mathrm{n}^{\circ}$ 4, 71-83.

LEVASSEUR L. \& CARDIN J.-F. (2012) : "The "social frameworks" of teaching high school history: teaching as part of the modernization of Québec society ». Journal of Social Science Education (JSSE), volume 11, number 4, 78-95.

Ministère de l'Éducation, du Loisir et du Sport (2007a) : Éthique et culture religieuse. Secondaire (Version approuvée) : Québec: Gouvernement du Québec.

Ministère de l'Éducation, du Loisir et du Sport (2007b) : Programme de formation de l'école québécoise. Enseignement secondaire, deuxième cycle. Québec: Gouvernement du Québec.

Ministère de l'éducation du Québec (1999) : Laïcité et religions. Perspective nouvelle pour l'école québécoise. Québec: Gouvernement du Québec.

RENAUT A. (2010) : Découvrir la philosophie, 2. La culture. Paris : Odile Jacob.

TAYLOR C. (2003) : La diversité de l'expérience religieuse aujourd'hui (traduit de l'anglais par J.-A. Billard). Montréal : Bellarmin.

TREMBLAY S. (2010) : École et religions. Genèse d'un nouveau pari québécois. Montréal : Fides.

WILlaIME J.-P. (2010) : Sociologie des religions. Paris: Presses universitaires de France. 